\title{
The Impact of COVID 19 on Oral Surgery Post- Operative Patient Complications and Communications Within the Oral Surgery Department at the Eastman Dental Hospital
}

\author{
Shree Patel, Sonita Koshal, and Onkar Mudhar
}

\section{ABSTRACT}

Introduction: A rise in post-operative complications (POCs) was observed following the second national lockdown due to the ongoing Coronavirus 2019 (COVID-19) pandemic. This prompted our Oral Surgery Department to investigate this to understand why this increase had occurred.

Aim: To evaluate the rise in POCs at The Eastman Dental Hospital Oral Surgery Department during and after the COVID-19 pandemic lockdown.

Materials and methods: A two cycle audit to assess the effect of the restrictions and ease imposed by lockdown on the number of POCs reported within the department.

Results: A spike in POCs was observed during the second lockdown. Following the ease of these restrictions, POCs reduced by half.

Discussion: As lockdown eased, the number of patient's contacting the department following surgical procedures significantly reduced. Did the pandemic cause patients to become more observant with regards to their post-operative site?

Conclusions: COVID-19 has had a lasting effect amongst our society and healthcare professionals are seeing not only the physical detriment but also the mental effects on our patients. We must ask if the increase in our POCs be caused by underlying "health anxiety" amongst members of the general public and if so, how can it be overcome?

Keywords: Anxiety, COVID-19 \& Post-operative Complications.
Published Online: November 09, 2021

ISSN: $2684-4443$

DOI: $10.24018 /$ ejdent.2021.2.6.107

Shree Patel*

The Eastman Dental Hospital, UK.

(e-mail: shree.patel3@nhs.net)

Sonita Koshal

Oral Surgery Consultant, The Eastman Dental Hospital, UK.

Onkar Mudhar

The Eastman Dental Hospital, UK.

*Corresponding Author

\section{INTRODUCTION}

Dental extractions are the most common type of procedure undertaken by Oral Surgery Departments. Indications for dental extractions include but are not limited to: pulpal or periapical pathology, periodontal disease, caries, pathology such as cysts or teeth within the fracture line following trauma.

Dental extractions are usually routine, and not as invasive as other treatments provided within the specialty; however post-operative complications (POCs) can still arise.

POCs due to dental extractions can range from $0-35 \%$ [1]. POCs differ between patients and can include post-operative pain and infection, alveolar osteitis (dry socket), paraesthesia of the inferior dental alveolar nerve and haemorrhage. The most common post-operative complication is dry socket, which is well documented in literature [2].

The Coronavirus pandemic originated as an epidemic in Wuhan, China. It is an infectious disease caused by the SARS-CoV-2 virus which rapidly spread globally from December 2019 to early 2020. It has not only caused detrimental effects to individual's physical health but also has had a significant impact on the amount of "health anxiety" individuals may suffer, with. Qiu et al. reporting that around $35 \%$ of people nationwide across China were psychologically affected by the COVID 19 pandemic. This statistic shows how significant the impact the virus was on mental health [3].

The main aim of this paper is to discuss our audit findings within the Oral Surgery Department of The Eastman Dental Hospital as to whether POCs following routine minor oral surgery procedures (MOS) had increased during the COVID19 Pandemic. This audit was initiated as the Department's clinicians noted an increase in patient communication during the second national lockdown following dental extractions.

Our department observed an increase of POCs during the second national lockdown (October 31st to December 2nd 2020), and subsequently a reduction when lockdown restrictions had eased within the United Kingdom. Prior to the lockdown POCs remained at an expected level. Ultimately, we wanted to analyse and understand if the pandemic had correlated with this observation. 


\section{COMMUNICATION AND SYSTEM IMPROVEMENTS}

This audit allowed us to identify barriers to communication between the patient and hospital after they had a procedure done within the Oral Surgery department. These highlighted areas were then assessed, and pathways constructed to improve the patient journey and allow easier reporting of post-operative complications within our department.

A recent change in our post-operative leaflets included adding an email address which allowed patients to email in their concerns or queries post-operatively. The previous communication entry was telephone only, but with staff working from home and changes to locations of clinics and offices this proved unreliable at the start of the pandemic.

The addition of an email address prior to the 5th audit cycle permitted the administration team to forward queries to a shared inbox. Clinicians would take on the role of triaging, followed by managing these concerns throughout the day. Patients were offered a face-to-face appointment if their signs and symptoms could not be managed remotely, such as dry socket, post-operative infections, numbness, and bony spicules. The email triage service proved to be an effective and efficient method of the patients receiving prompt dental advice.

Following the COVID pandemic, a rise in post-operative complications and patient communication were noted amongst the clinicians. Hence the audit was initiated to look into the causes of this observation. Prior to this, 3 audit cycles were undertaken and post-operative complication rates can be seen in Fig. 1. A clear increase is noted from cycle 3 to cycle 4, which corresponds to the Government's restrictions.

\section{Mean Post-Operative Complication Rate}

(\%)

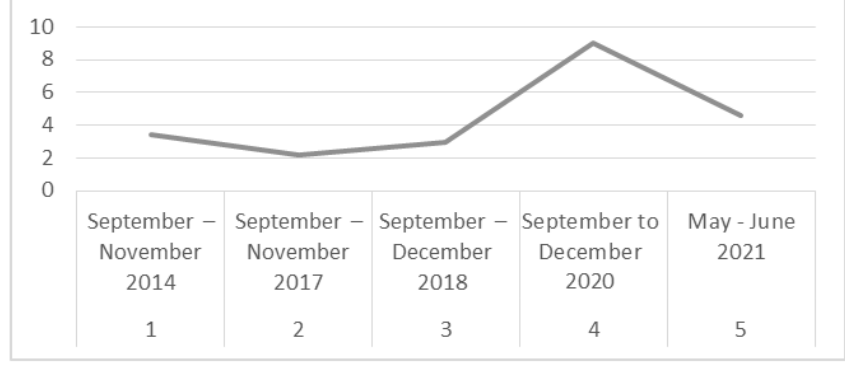

Fig. 1. A visual representation of results from all audit cycles undertaken.

\section{Covid-19 EfFects On Mental Health AND ANXIETy}

Millions of people have suffered the effects of COVID-19. There is evidence exploring the effects of how COVID-19 has caused both physical and mental consequences to individuals.

The Government's rules of restriction lead many to feel trapped in an environment with little to distract them. This led to an increase in anxiety, fear and focus on health in particular, as this was deemed "at risk".

The health anxiety was further compounded by the reduction and adaptation of regular services, due to staff and patient risk and redeployment. This led to the population feeling they did not have the same level of support, which had a compounding effect along with having more time to think about one's health.
Within dentistry, the risk of aerosol generating procedures meant that dental practices were closed for prolonged periods of time, gradually reopening with an increase in precautions and concern. Thankfully this has now improved and those in primary care are operating at the levels seen pre-pandemic.

More recent literature has explored the effects of COVID19 on the public's mental health. A systematic review by Vindegaard and Benros [4] explored the effects of COVID19 on the general public. Sonderskov et al. [5] observed a lower psychological well-being (WHO-5) following COVID19. Li et al. [6] also noted an increase of words that were emotional indicators of anxiety and depression on a popular Chinese social media site post-COVID [4]-[6]. These studies, amongst others, show that COVID has had an enormous effect on the mental wellbeing on our society.

Literature discussing the link between anxiety and COVID-19 is still limited, however Holmes et al. [7] have highlighted that mental health is an important topic to research further.

\section{METHOD}

A two-cycle audit was undertaken within the Oral Surgery Department at The Eastman Dental Hospital from 2020 to 2021. This was carried out to assess the recent lockdown's effect on the rise of POCs. These were the 4th and 5th audit cycles (Table I). Previous audit cycles before the pandemic maintained a low post-operative complication rate, with an average of over 3 cycles of $2.85 \%$.

All audit cycles had a standard based on literature and local commissioner's guidelines with a POCs rate of around 4-5\%. Sampling for all cycles included a random sample of patients that attended for a MOS procedure within our department under local anaesthetic, intravenous sedation, or general anaesthetic. The type of procedure, mode of anaesthesia, clinician's grade (which was reflective of their experience), complications and management for each patient were recorded.

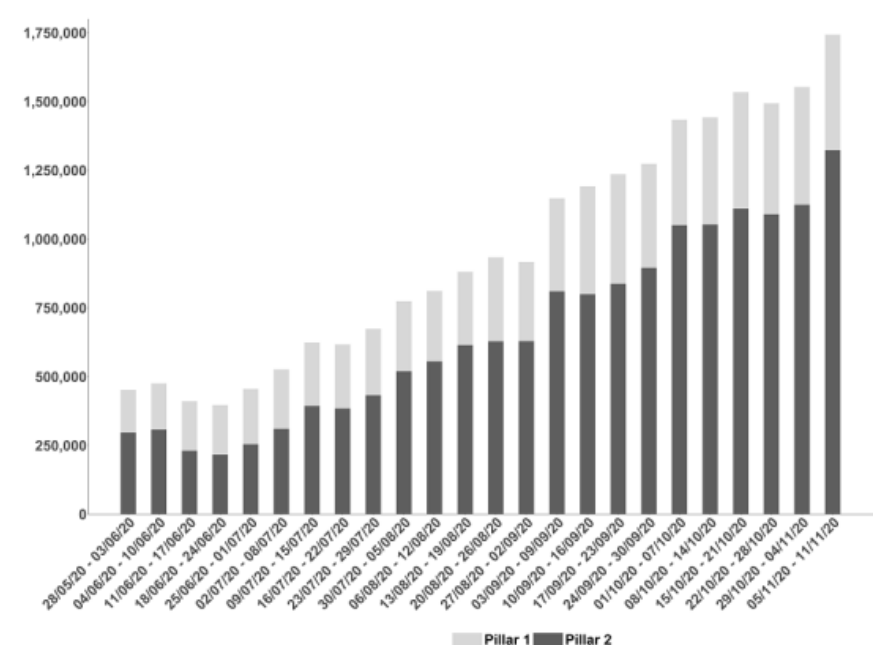

Fig. 2. Directly taken from Gov.UK website. This graph shows the number of people who tested positive for COVID-19 at weekly intervals. We can see the rise from September to November 2020 being the highest number of positive cases. This corresponds to the spike in our POCs [8]. 
TABLE I: RESULTS FROM ALL AUdIT CYCLES UNDERTAKEN WITH MEAN POST-OPERATIVE COMPLICATIONS

\begin{tabular}{ccc}
\hline $\begin{array}{c}\text { Audit } \\
\text { Cycle }\end{array}$ & Time Period & $\begin{array}{c}\text { Mean Post-Operative } \\
\text { Complication Rate (\%) }\end{array}$ \\
\hline 1 & September - November 2014 & 3.43 \\
2 & September - November 2017 & 2.19 \\
3 & September - December 2018 & 2.93 \\
4 & September - December 2020 & 9 \\
5 & May-June 2021 & 4.56 \\
\hline
\end{tabular}

\section{RESUlTS}

For the purpose of this paper, we will focus on audit cycles 4 and 5 to assess the effect of lockdown on our post-operative complication rate. Cycle 4 had almost 4 times more POCs compared to its previous cycle result as seen in Table I. Cycle 3 was prior to the pandemic. Cycle 4's time frame includes the second lockdown where the majority of the general public were situated at home. In contrast, cycle 5 shows a reduction in POCs by nearly $50 \%$, which may be in part be due to the ease of lockdown restrictions and feeling of "freedom" returning.

For Cycle 4 and 5 the most frequent complication was dry socket. The second most common POC was an infected socket. Cycle 4 has $55 \%$ patients diagnosed with dry socket as opposed to $3.125 \%$ in cycle $5.33 \%$ of patients in cycle 4 had post-operative infections as opposed to $1 \%$ in cycle 5 .

A wide range of staff members were included in both audit cycles but there was no clear correlation between job role and incidence of POCs.

In Cycle 5, 6 patients were recorded as having normal postoperative healing. Yet the clinical notes show these patients had reported pain or concern of their post-operative site.

The results have made us question if they are reflective of the pandemic. We must consider if the rise in POCs in cycle 4 is due to patients having more time to think about the extraction site and therefore be more concerned than usual due to fewer distractions such as work or not being able to go outside without restrictions. Another factor to consider is the possible increased anxiety or fear instilled by the pandemic.

\section{DISCUSSION}

The Eastman Dental Hospital maintained a high level of surgical activity throughout these time periods, working with extra precautions and care as required.

The height of the pandemic showed a large increase in post-operative complications, and the most recent audit results showed a reduction in this number.

This pattern corresponds to the ease of COVID-19 lockdown restrictions which began on the 8th of March 2020. This indicates that the pandemic restrictions potentially had an effect on patient's anxiety and resulted in an increase in contact to us, which on the 5 th cycle has now begun to return to the average and expected levels.

Several patients would email in with a photograph of their extraction site and describe what they thought they could see, often it was normal healing anatomy. We question if this could be due to patient's being at home working, on furlough or restricted, resulting in having more time to think about their extraction site.

Another hypothesis for the increase in actual dry socket and infections on the 4th cycle is also "over care" of the surgical site. If patients are too focused on the site, they may be overly rinsing, brushing, and tampering with the region out of habit and boredom. It is well known that dry socket can occur when the blood clot is "washed away".

The department also felt the addition of the triage email address on our post-operative leaflet was a positive change and led to an increase of clear and specific patient concerns. This was a simple and effective method for patients to communicate with the team and solved a historic issue.

The analysis of the number of POCs, led the team to consider whether patients could be given more verbal instructions of what to expect following a MOS, with more specific and general care information given. This may include to not over-focus on the surgical sites, reassurance, and repetition of what we consider "normal" healing. Patients are already given leaflets prior to their procedure, and these were reviewed, piloted, and confirmed as sufficient.

Another thought to consider is whether the POC number is returning to average or if it is still high and that the extra measures, we put in place are counteracting this at the moment. We will need to repeat this cycle again to confirm if there has been a reduction in POCs since the pandemic.

\section{CONCLUSION}

Dental anxiety is well known, and a frequent emotion felt by patients [8]. Our results following this two-audit cycle reflect on the effects of the pandemic on the number of POCs received. An increase of POCs during enforcement of lockdown restrictions may indicate that patients had more time to think about their post-operative site, developing anxiety, or even inadvertently tampering with the site, creating a complication. A re-audit will be undertaken between September to December 2021 to assess the POC rate in the department. We will re-analyse the percentage and assess if it within the normal departmental limit.

By undertaking this audit, it has allowed our team to understand our patient base and identify the main causes for our POCs. This has ultimately led us to improve upon and provide robust patient care.

\section{REFERENCES}

[1] Dallaserra M, Poblete F, Vergara C, Cortés R, Araya I, Yanine N, Villanueva J., Infectious postoperative complications in oral surgery. An observational study, Journal of clinical and experimental dentistry, 2020 Jan;12(1):e65.

[2] Rakhshan V., Common risk factors for postoperative pain following the extraction of wisdom teeth, Journal of the Korean Association of Oral and Maxillofacial Surgeons, 2015 Apr 1;41(2):59-65.

[3] Qiu J., Shen B., Zhao M., Wang Z., Xie B., Xu Y., A nationwide survey of psychological distress among Chinese people in the COVID-19 epidemic: implications and policy recommendations, General psychiatry, 2020;33(2).

[4] Vindegaard N, Benros M. E., COVID-19 pandemic and mental health consequences: Systematic review of the current evidence, Brain, behavior, and immunity, 2020 Oct 1;89:531-42.

[5] Sønderskov K. M., Dinesen P. T., Santini Z. I., Østergaard S. D., The depressive state of Denmark during the COVID-19 pandemic, Actaneuropsychiatrica, 2020 Aug;32(4):226-8.

[6] Li S., Wang Y., Xue J., Zhao N., Zhu T., The impact of COVID-19 epidemic declaration on psychological consequences: a study on active Weibo users, International Journal of Environmental Research and Public Health, 2020 Jan;17(6):2032. 
[7] Holmes E. A., O'Connor R. C., Perry V. H., Tracey I., Wessely S., Arseneault L., Ballard C., Christensen H., Silver R. C., Everall I., Ford T., Multidisciplinary research priorities for the COVID-19 pandemic: a call for action for mental health science, The Lancet Psychiatry,2020 Jun 1;7(6):547-60.

[8] Weekly statistics for NHS Test and Trace (England) and coronavirus testing (UK): 5 November to 11 November [Internet]. GOV.UK. 2021 [cited 14 October 2021]. Available from: https://www.gov.uk/government/publications/nhs-test-and-traceengland-and-coronavirus-testing-uk-statistics-5-november-to-11november/weekly-statistics-for-nhs-test-and-trace-england-andcoronavirus-testing-uk-5-november-to-11-november.

[9] Appukuttan D. P., Strategies to manage patients with dental anxiety and dental phobia: literature review, Clinical, cosmetic and investigational dentistry, 2016;8:35 